(C) 2017 IEEE. Personal use of this material is permitted. Permission from IEEE must be obtained for all other uses, in any current or future media, including reprinting/republishing this material for advertising or promotional purposes, creating new collective works, for resale or redistribution to servers or lists, or reuse of any copyrighted component of this work in other works. 


\title{
Low-Complexity Uplink Multiuser Receivers for MIMO System with Massive Hybrid Array
}

\author{
Hang Li, Thomas Q. Wang, Xiaojing Huang, and Jian A. Zhang \\ Global Big Data Technologies Center (GBDTC), University of Technology, Sydney, Australia \\ \{Hang.Li, Qian.Wang, Xiaojing.Huang, Andrew.Zhang\}@uts.edu.au
}

\begin{abstract}
Due to the enormous needs for signal processing and hardware constraints, the full digital implementation for a large antenna array at mmWave frequencies becomes intractable. Hence, receiver design for MIMO system with massive hybrid array is very demanding, particularly for subarray structure. In this paper, we propose two low-complexity uplink multiuser receiver design schemes (single-beam and multi-beam) under the circumstances of only users' angles of arrival (AOAs) available at base station, which greatly simplify the analog beamforming structure and reduce the complexity of channel estimation especially when the number of antenna elements is considerably large. In the single-beam scheme, subarrays are mapped to different users one by one such that each subarray serves a specific user. In the multibeam scheme, all subarrays use the same analog beamforming and any subarray's beamforming signal potentially points at all users. Digital beamforming is then employed to combine all subarray signals followed by equalization. Simulations are performed to compare the proposed schemes with other schemes that need channel information available at the subarray output. Since only AOAs are required for analog and digital beamformings, the proposed schemes are more suitable for mmWave MIMO system with massive hybrid array in spite of slight sum-rate loss.
\end{abstract}

\section{INTRODUCTION}

To compromise between the system performance and hardware cost, millimeter wave (mmWave) beamforming with massive hybrid antennas array [1]-[3] has been regarded as an enabling technology for $5 \mathrm{G}$ wireless communication systems. A typical hybrid array architecture consists of multiple analog beamformers and multiple radio-frontend (RF) chains, and each analog beamformer includes multiple adjustable analog phase shifters and/or sometimes analog attenuators. According to the mapping from antenna elements to RF chains, which requires different number of phase shifters, hybrid array architecture can be categorized into the fully-connected structure [2] where each antennas connects to multiple phase shifters and all RF chains, and the subarray structure [1] where each antenna only connects to one phase shifter and one RF chain. The fullyconnected structure employs full beamforming gain for each RF chain such that better performance can be achieved. However, it requires too many phase shifters particularly for massive antennas, thus greatly increasing the hardware implementation complexity. On the other hand, the subarray structure, that uses much less phase shifters by connecting each RF chain only with part of the antennas, is preferable for practical hardware

This research was supported under Australian Research Council's Discovery Projects funding scheme (project number DP160101693). and physical deployment with massive antennas at the cost of tolerable performance loss.

Owing to the hybrid array architecture with analog and digital precoding/combining that provides great flexibility for beamforming design, it requires either independent or joint precoding/combining algorithms [4] to optimize the system performance. Reasonably, the system capacity with hybrid array is determined by equivalent baseband channels, which includes actual physical channels and RF beamforming. RF beamforming generally adopts discrete analog phase shift and attenuate values, which makes optimization problems very challenging. So far, some relevant works using approximation and suboptimization methods have been presented in the literature for the fully-connected structure [5], [6] and subarray structure [7][9], but the optimal or near-optimal solutions for the latter are still being explored as yet.

In the following, we focus reviews on the subarray structure schemes presented in the literature. In [7], a multi-beam transmission diversity scheme based on hybrid subarray structure is proposed and investigated for single user MIMO with single data stream. It greatly exploits the flexibility by adaptively combining the analog beamforming and digital precoder. The work in [8] studies a near-optimal iterative hybrid precoding scheme for single user MIMO with multiple data streams, where the complicated capacity optimization problem is decomposed into a series of sub-optimal problems, and the achievable capacity of each subarray is optimized one by one using the idea of successive interference cancellation. Recently, the authors in [9] propose some low-complexity user-directed RF beamforming schemes for multiuser mmWave MIMO systems, where the channel correlation between different users can be efficiently reduced by allocating different subarrays to different users. Simulation results in [9] compare the proposed schemes with other representative schemes, and provide insights on beamforming design for the subarray structure. Although the above schemes achieve good performance, channel information at each antenna element is required to be known, which potentially increases the number of channel estimation with the increasing number of antennas.

In this paper, we propose two low-complexity uplink multiuser receiver design schemes 1) single-beam scheme and 2) multi-beam scheme, where only users' angles of arrival (AOAs) are required at the base station (BS), which makes analog beamforming implementation easy and also avoids substantial channel estimation induced by a large number of antennas. In 
the single-beam scheme, the basic idea is to allocate subarrays to users one by one such that each subarray's beamforming signal points at a specific user, whereas in the multi-beam scheme, to allocate different subarrays with the same analog beamforming weights such that any subarray's beamforming signal potentially points at all users. Followed by an equalizer, a digital beamformer with only AOA information is used to combine all subarray signals to produce the final beamforming signals. Simulation results are provided for comparison between the proposed schemes and other schemes that need known channel information at the subarray output. The proposed schemes that only require AOA information provides a good balance between the performance and implementation complexity for mmWave MIMO system with massive hybrid array.

The following notations are used throughout this paper. A, $\mathbf{a}$ and $a$ stand for a matrix, a column vector and a scalar, respectively; $\mathbf{A}_{i, j}$ is the entry on the $i$ th row and $j$ th column of $\mathbf{A} ; \mathbf{A}^{T}$ and $\mathbf{A}^{H}$ denote the transpose and conjugate transpose of $\mathbf{A}$, respectively. $\|\mathbf{a}\|_{2}$ is the 2-norm of $\mathbf{a}$, and $\mathbf{I}_{N}$ is the identity matrix with $N$ dimensions; $\mathcal{C N}(\mathbf{m}, \mathbf{V})$ represents a complex Gaussian random vector with mean $\mathbf{m}$ and covariance matrix V. Further, the notations $\log (\cdot)$ and $|(\cdot)|$ represent the logarithmic and determinant or absolute value depending on context of $(\cdot)$, respectively.

\section{Problem Formulation}

\section{A. System Model}

Consider a narrowband multiuser uplink transmission system, which consists of a BS with a hybrid array [1] and $K$ users, each with a single omnidirectional antenna. As shown in Fig.1, the receiver at the BS is assumed to use $M(M \geq K)$ analog subarrays each with $N$ antenna elements. Each analog subarray adjusts the signal phase and attenuation at each element using analog phase shifter and attenuator, then combines the signals with an analog mixer and downconverts to generate a complex baseband signal, which is called analog beamforming. Following the outputs of analog beamformer, digital beamforming is performed to combine all subarray signals to produce the final beamformed signal. Finally, an equalizer is applied to mitigate multiuser interference. Thanks to very high carrier frequency in mmWave systems, large arrays can be possibly implemented in a compact size. In general, $N$ is far larger than 1 , thereby significantly reducing the number of digital chains.

We denote the transmitted signals and powers of $K$ MSs as $K \times 1$ vector of $\mathbf{s}=\left[s_{0}, s_{1}, \ldots, s_{K-1}\right]^{T}$, where $\mathbf{s} \sim \mathcal{C N}(\mathbf{0}, \mathbf{P})$ and $\mathbf{P}=\operatorname{diag}\left\{p_{0}, p_{1}, \ldots, p_{K-1}\right\}$, respectively. Let $\mathbf{H}_{m}=$ $\left[\mathbf{h}_{m, 0}, \mathbf{h}_{m, 1}, \ldots, \mathbf{h}_{m, K-1}\right]$, where $\mathbf{h}_{m, k}$ is the $N \times 1$ channel vector between MS $k$ and subarray $m$ of the BS. The received signal vector $\mathbf{y}_{m}$ at subarray $m$ can be expressed as

$$
\mathbf{y}_{m}=\mathbf{H}_{m} \mathbf{s}+\mathbf{n}_{m}
$$

where $\mathbf{n}_{m} \sim \mathcal{C N}\left(\mathbf{0}, \sigma^{2} \mathbf{I}_{N}\right)$ denotes the $N \times 1$ additive white Gaussian noise at subarray $m$. By stacking $\mathbf{y}_{m}$ one by one, we

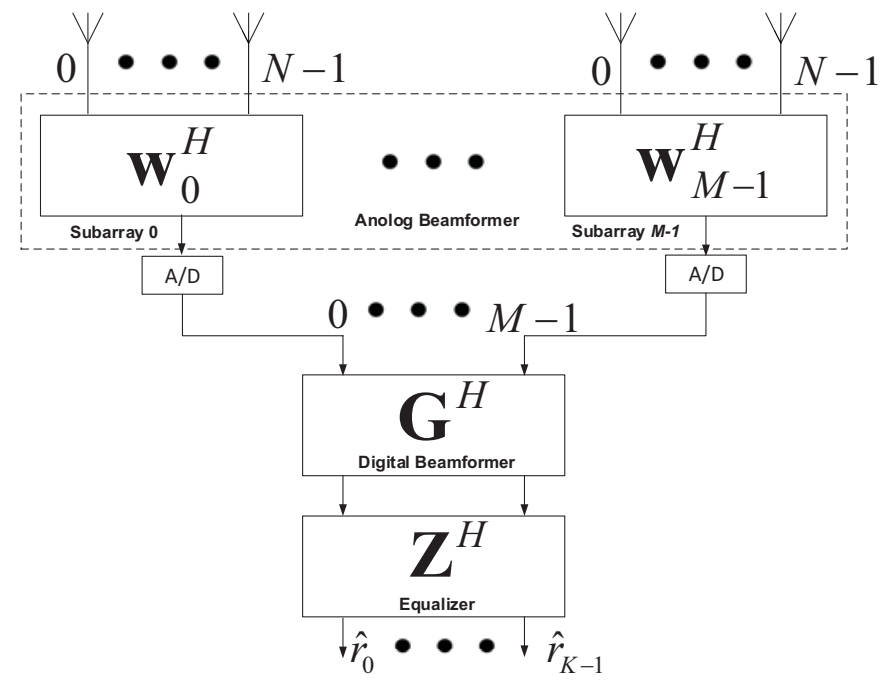

Fig. 1. Block diagram of an uplink multiuser MIMO receiver with hybrid array

have the received signal vector $\mathbf{y}=\left[\mathbf{y}_{0}^{T}, \mathbf{y}_{1}^{T}, \ldots, \mathbf{y}_{M-1}^{T}\right]^{T}$ at the BS

$$
\mathbf{y}=\mathbf{H s}+\mathbf{n},
$$

where $\mathbf{H}=\left[\mathbf{H}_{0}^{T}, \mathbf{H}_{1}^{T}, \ldots, \mathbf{H}_{M-1}^{T}\right]^{T}$, and $\mathbf{n}=$ $\left[\mathbf{n}_{0}^{T}, \mathbf{n}_{1}^{T}, \ldots, \mathbf{n}_{M-1}^{T}\right]^{T}$.

Let $\mathbf{W}$ be the $N M \times M$ analog beamforming matrix, which can be represented by

$$
\mathbf{W}=\left(\begin{array}{cccc}
\mathbf{w}_{0} & \mathbf{0} & \cdots & \mathbf{0} \\
\mathbf{0} & \mathbf{w}_{1} & \cdots & \mathbf{0} \\
\vdots & \vdots & \ddots & \vdots \\
\mathbf{0} & \mathbf{0} & \cdots & \mathbf{w}_{M-1}
\end{array}\right),
$$

where $\mathbf{w}_{m}$ is an $N \times 1$ analog weight vector of subarray $m$ with $\left\|\mathbf{w}_{m}\right\|_{2}=1$, and $\mathbf{0}$ is an $N \times 1$ vector with all elements 0 .

Let $\mathbf{G}=\left[\mathbf{g}_{0}, \mathbf{g}_{1}, \ldots, \mathbf{g}_{K-1}\right]$ be an $M \times K$ digital beamforming matrix, and the equivalent baseband channel matrix between the users and the beamforming output of the BS can be defined as $\mathbf{G}^{H} \mathbf{W}^{H} \mathbf{H}$. Assuming that the channel state information is perfectly available at the BS, the achievable uplink sum-rate of the system is given by

$$
\begin{aligned}
R & =\log _{2}\left|\mathbf{I}_{K}+\mathbf{Q}^{-1} \mathbf{G}^{H} \mathbf{W}^{H} \mathbf{H} \mathbf{P} \mathbf{H}^{H} \mathbf{W G}\right| \\
& =\log _{2}\left|\mathbf{I}_{K}+\frac{1}{\sigma^{2}}\left(\mathbf{G}^{H} \mathbf{G}\right)^{-1} \mathbf{G}^{H} \mathbf{W}^{H} \mathbf{H} \mathbf{P} \mathbf{H}^{H} \mathbf{W G}\right|,
\end{aligned}
$$

where $\mathbf{Q}=\sigma^{2} \mathbf{G}^{H} \mathbf{W}^{H} \mathbf{W G}$ is the covariance matrix of noise.

\section{B. Channel Model}

Throughout the paper, we consider a narrowband channel representation $\mathbf{h}_{m, k}$ as follows

$$
\mathbf{h}_{m, k}=\sum_{p=0}^{P-1} \alpha_{k, p} \mathbf{a}_{m}\left(\phi_{k, p}\right),
$$

where $P$ is the number of paths, and $\alpha_{k, p}$ and $\phi_{k, p}$ stand for the channel complex gain and AOA of path $p$ for user $k$, 
respectively. $\mathbf{a}_{m}\left(\phi_{k, p}\right)$ is the subarray $m$ response vector to the $p$ th path signal with AOA $\phi_{k, p}$.

Without loss of generality, we assume that each subarray consists of isotropic elements with omni-directional radiation patterns, i.e., the radiation pattern in any angle is one. In this paper, we consider the uniform linear array (ULA) of localized subarrays, although the proposed schemes are also applicable for planar array or interleaved subarrays. Therefore, $\mathbf{a}_{m}\left(\phi_{k, p}\right)$ can be written as

$$
\mathbf{a}_{m}\left(\phi_{k, p}\right)=e^{j \frac{2 \pi d}{\lambda} N m \sin \phi_{k, p}} \mathbf{a}_{0}\left(\phi_{k, p}\right),
$$

where $\lambda$ is the carrier wavelength, $d$ is the adjacent element spacing and $\mathbf{a}_{0}\left(\phi_{k, p}\right)$ is an $N$-dimensional column vector with the $n$th $(n=0,1, \ldots, N-1)$ element of $e^{j \frac{2 \pi d}{\lambda} n \sin \phi_{k, p}}$.

\section{LOW-COMPLEXITY RECEIVER DESIGN WITH MASSIVE HYBRID ARRAY}

Mmwave channel model is better characterized by spatially sparse multipath channel [10], where the line-of-sight (LOS) path dominates the power distribution across the multipath. Especially when the ratio $\eta$ of the power of the dominating LOS path to sum power of the remaining paths is large, it can be assumed that the AOA of LOS signal is approximately regarded as the AOA of multipath signals, which greatly simplifies signal processing.

The full channel knowledge between each antenna element (or subarray) and each user is generally required for most of schemes, which greatly increases the complexity of channel estimation especially when massive hybrid array is used. To reduce the complexity, we propose single-beam and multi-beam schemes that only requires AOA information available.

\section{A. Single-beam scheme with known AOA}

Since only AOA information is available, and users' channel magnitude response for each element is unknown, the optimization of the mapping from subarrays to users are not considered in this paper. We allocate subarrays to users successively following $\mathbf{w}_{m}=\mathbf{a}_{0}\left(\phi_{m \bmod K}\right) / \sqrt{N}$, where $m \bmod K$ is the remainder of the division of $m$ by $K$ and $\phi_{k}$ is the AoA of the LOS path for user $k$. Hence, the $m$ th subarray's beamforming signal directs at the $(m \bmod K)$ th user, which effectively reduces the channel correlation between different users.

Due to parts of subarrays directing at the same user, we combine the corresponding subarray output signals for different users in digital beamforming using the following $\mathbf{G}$,

$$
\mathbf{G}_{m, k}= \begin{cases}\sqrt{\frac{K}{M}} e^{j \frac{2 \pi d}{\lambda} N m \sin \phi_{k}}, m=k, k+K, k+2 K, \ldots \\ 0, & \text { otherwise }\end{cases}
$$

By substituting $\mathbf{w}_{m}$ and (6) into (3), we obtain the system sumrate.

\section{B. Multi-beam scheme with known AOA}

In the multi-beam scheme, we aim at directing at all users by generating multi-beam analog beamforming. Reasonably, we sum all subarray response vectors $\mathbf{a}_{0}\left(\phi_{k}\right)$ with respect to $k$, and then normalize it as $\mathbf{w}_{m}, m=0,1, \ldots, M-1$,

$$
\mathbf{w}_{m}=\frac{\sum_{k=0}^{K-1} \mathbf{a}_{0}\left(\phi_{k}\right)}{\left\|\sum_{k=0}^{K-1} \mathbf{a}_{0}\left(\phi_{k}\right)\right\|_{2}}
$$

where the $k$ th term in the numerator makes one of the multibeams direct to the $k$ th user, while inducing the interferences to other users, which is expected to be removed in equalization part.

Since all subarrays contribute to directing at any user, we combine all subarray output signals for user $k$ in digital beamforming as

$$
\mathbf{g}_{k}=\left[1, e^{j \frac{2 \pi d}{\lambda} N \sin \phi_{k}}, \ldots, e^{j \frac{2 \pi d}{\lambda} N(M-1) \sin \phi_{k}}\right]^{T} / \sqrt{M} .
$$

It is noted here that although the channel correlation between different users is not reduced, the multi-beam scheme greatly simplifies the analog implementation due to single configuration and boosts the expected signals by beamforming.

\section{The schemes with known channel}

In the following, we consider some schemes with channel information available at the subarray output. Without loss of generality, let the digital beamforming matrix $\mathbf{G}=\mathbf{W}^{H} \mathbf{H}$, which requires channel information at the subarray output available instead of AOA information. We adopt different analog beamforming methods for the following schemes.

1) Single-beam and Multi-beam schemes with known channel: For analog beamforming, the same weights as the proposed ones are used, while different digital beamforming weights that depend on channel information at the subarray output are generated. Hereinafter, it is not specifically detailed.

2) The upper-bound with optimal linear combinations: With only AOA information available, we assume that there exists an optimal linear combination of subarray response vector $\mathbf{a}_{0}\left(\phi_{k}\right)$ with respect to $k$ as $\mathbf{w}_{m}$ to maximize the sum-rate. However, the linear combination coefficients are practically hard to obtain as a result of the excessive search scope. Therefore, we adopt the switches ( 0 or 1$)$ as on/off linear combination coefficients to greatly reduce search scope. In such cases, the optimal linear combinations can be obtained by

$$
\hat{\mathbf{w}}_{m}=\underset{\mathbf{w}_{m}^{l}}{\operatorname{argmax}} R,
$$

equivalently, where

$$
\mathbf{w}_{m}^{l}=\frac{\sum_{k \in \Omega_{l}} \mathbf{a}_{0}\left(\phi_{k}\right)}{\left\|\sum_{k \in \Omega_{l}} \mathbf{a}_{0}\left(\phi_{k}\right)\right\|_{2}}
$$

and $\Omega_{l} s, l=1,2, \ldots, 2^{K}-1$, denote all non-empty subsets of $\Omega=\{1,2, \ldots, K\}$.

By switching on/off users' subarray response vectors as shown in (10), this scheme tries $\left(2^{K}-1\right)^{M}$ linear combinations to obtain performance upper-bound of the proposed ones. It is obviously seen that analog beamforming vector $\mathbf{w}_{m}$ in Section 
III $A$. and $B$. are two special cases in search sets, where the cardinality of $\Omega$ is 1 and $K$, respectively.

3) The Eig-beamforming scheme: To maximize the received signal sum power from all AOA directions, the optimal solution would be the eigen-beamforming, and $\mathbf{w}_{m} s, m=0,1, \ldots, M-1$, would be the eigen-vector corresponding to the maximal eigenvalue of $\mathbf{B B}^{H}$, where $\mathbf{B}=$ $\left[\mathbf{a}_{0}\left(\phi_{0}\right), \mathbf{a}_{0}\left(\phi_{1}\right), \ldots, \mathbf{a}_{0}\left(\phi_{K-1}\right)\right]$. In other words, $\mathbf{w}_{m}$ is one column (Its index is the maximal diagonal element index of D.) of unitary matrix $\mathbf{U}$ in eigen-decomposition of $\mathbf{B B}{ }^{H}=\mathbf{U D U}^{H}$.

Although the signal sum power can be maximized using the Eig-beamforming scheme, the reduction of channel correlation for different users is not taken into account. Hence, the achieved sum-rate is very low as shown in simulation results to be presented.

\section{Simulation Results}

The proposed schemes are simulated using a hybrid ULA of localized subarrays. $d$ is set to be $\lambda / 2$. We assume that each user experiences multipath fading channel in cluster, and in each cluster the number of multipath is 6 with one LOS-path and the remaining NLOS-paths. The channel fading coefficients are generated following a complex Gaussian distribution with zero mean, and the ratio $\eta$ of the variances used for LOSpath to the sum of NLOS-paths. The AoAs of the $k$ th user's signal from NLOS-paths are uniformly distributed in the range of $\left[\phi_{k}-\delta, \phi_{k}+\delta\right]$, and $\left\{\phi_{k}\right\} s, k=0, \ldots, K-1$ are generated with a uniform distribution over $\left[-90^{\circ}, 90^{\circ}\right]$. Unless otherwise specified, the default values used in simulations are as follows: the number of subarrays $M=16$, the number of antennas in each subarray $N=8$, the number of users $K=4, \eta=10 \mathrm{~dB}$, $\delta=5^{\circ}$ and the SNR per antenna is $16 \mathrm{~dB}$. The sum-rate performance of upper-bound is obtained by exhaustive search. The average bit error rate (BER) performance with QPSK modulation and zero-forcing $(\mathrm{ZF})$ equalization is evaluated for all users.

Fig. 2 shows the sum-rate bps/Hz versus the received SNR per antenna. The sum-rate increases along with the SNR, and the scheme with upper-bound outperforms the others as expected. When only AOA information is known, the single-beam scheme performs better performance than multi-beam scheme with about $5.7 \%$ sum-rate gain. The proposed single-beam and multi-beam schemes incur $2.2 \%$ and $1.3 \%$ sum-rate loss compared with the corresponding ones with known channel, respectively. Meanwhile, they only require $K=3$ AOAs instead of $M=6$ channel information at subarray output. The Eig-beamforming scheme is inferior to the proposed schemes due to no consideration of reducing channel correlation for different users, although it maximizes the output signal power. The conventional MIMO scheme has the worst performance as no subarray analog beamforming signals are combined, where the number of antennas is assumed to be $M$.

Fig. 3 shows the sum-rate $\mathrm{bps} / \mathrm{Hz}$ versus the number of subarrays. It is easily shown that when the number of subarrays $M$ is smaller than 16, the proposed single-beam scheme outperforms multi-beam scheme in terms of sum-rate, while the

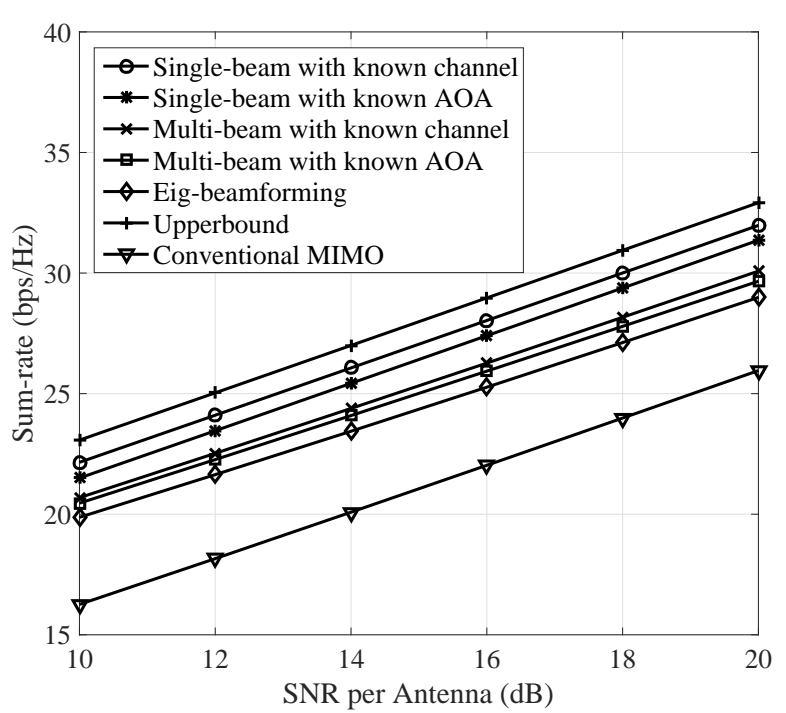

Fig. 2. Sum-rate versus the SNR per antenna, where $M=6, N=8$ and $K=3$.

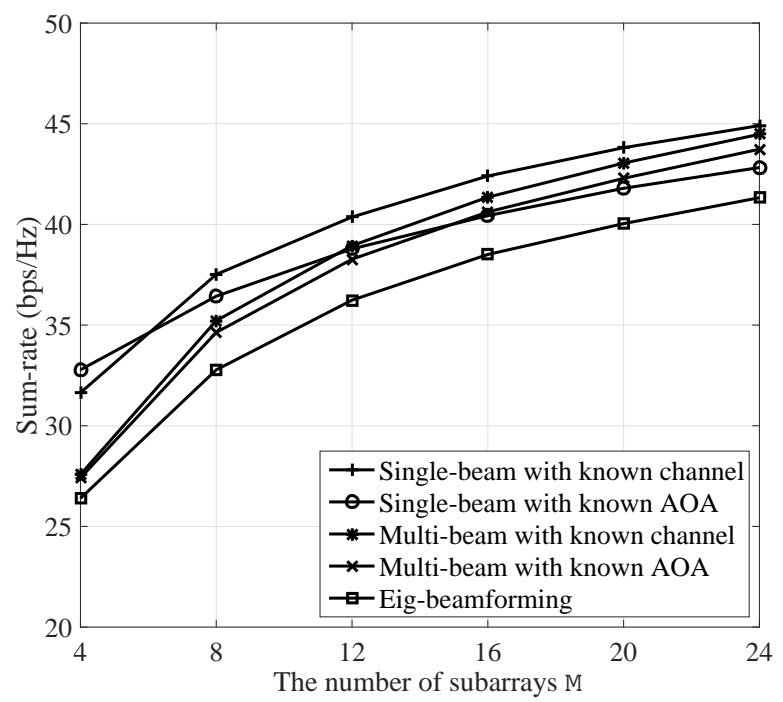

Fig. 3. Sum-rate versus the number of subarrays $M$.

proposed multi-beam scheme has better performance when $M$ is over 16. According to different numbers of subarrays, they provide flexible receiver structures to achieve higher sum-rate. In addition, the two schemes with known channel achieve close performance when $M$ is reasonably large.

Fig. 4 shows the sum-rate $\mathrm{bps} / \mathrm{Hz}$ versus the number of antennas in each subarray. From Fig. 4, we can see that the proposed multi-beam scheme presents better performance than that of single-beam when the number of antennas in each subarray $N$ is below 10 . On the other hand, the proposed singlebeam scheme outperforms multi-beam scheme when $N$ is over 10. Likewise, either of the proposed schemes can be selected to achieve higher sum-rate following different number of antennas in each subarray. The single-beam scheme with known channel always exceeds the multi-beam scheme regardless of $N$.

Fig. 5 demonstrates the impact of different path-power-ratio 


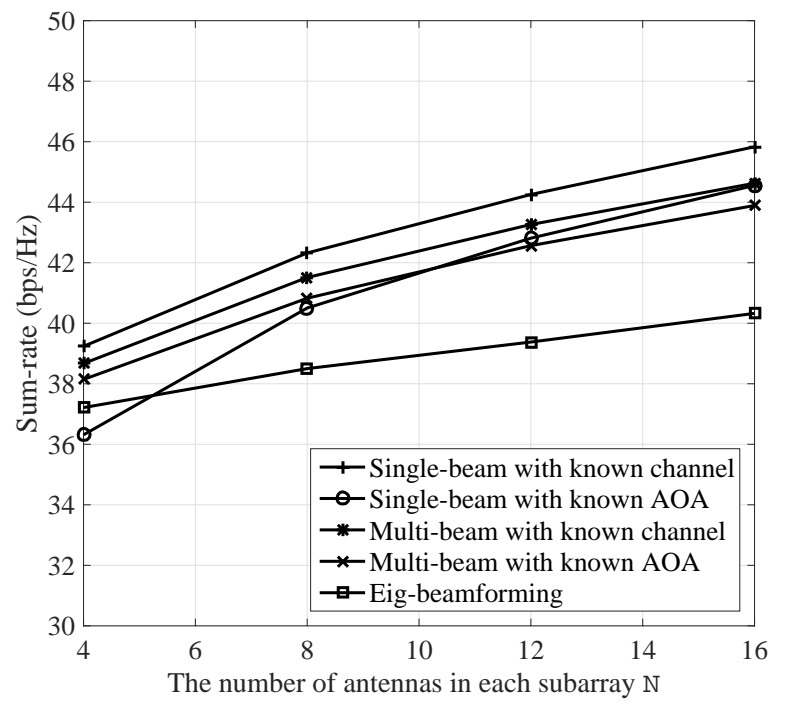

Fig. 4. Sum-rate versus the number of antennas in each subarray.

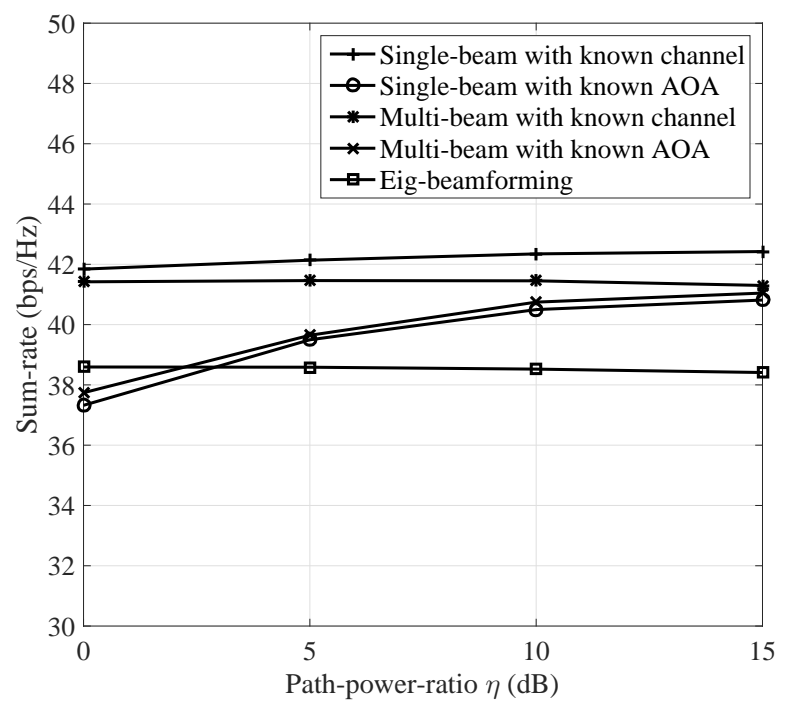

Fig. 5. Sum-rate versus the path-power-ratio $\eta$.

$\eta$ on sum-rate bps/Hz. It is obviously shown that the proposed two schemes have higher sum-rate as $\eta$ increases, while the other schemes are not sensitive to different $\eta$. It indicates that our proposed schemes are more applicable to the typical mmWave LOS-path dominating scenarios.

Fig. 6 shows BER versus the received SNR per antenna. The proposed single-beam scheme performs far better BER performance than that of multi-beam scheme, which is because single-beam scheme reduces the channel correlation between different users on analog bemaforming part. It also suggests that $\mathrm{ZF}$ equalization is inappropriate for the latter, and the advanced equalization methods are supposed to be exploited in receiver designs, which can be an open problem.

\section{CONCLUSION}

In this paper, we have presented two low-complexity uplink multiuser receiver design schemes for mmWave massive hybrid

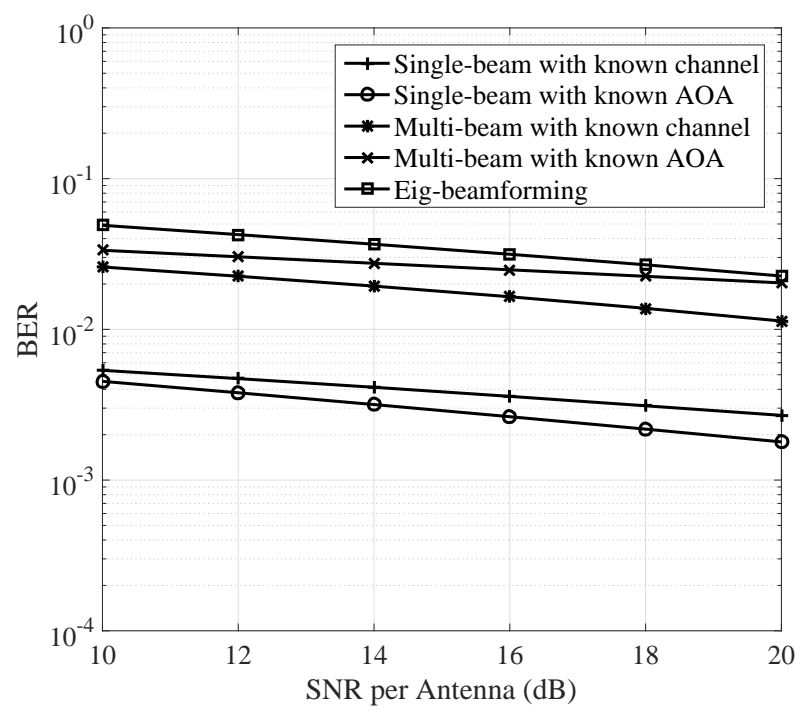

Fig. 6. BER versus the SNR per antenna, where $\eta=20 \mathrm{~dB}$.

array with the subarray structure, which are particularly applicable to the scenario for only users' AOA information available. The proposed schemes can simplify analog beamforming structure and avert the channel estimation for each antenna element. Simulation results are provided for comparison among all schemes in terms of sum-rate and BER. The proposed schemes that only require AOA information achieve good performance at low complexity.

\section{REFERENCES}

[1] J. Zhang, X. Huang, V. Dyadyuk, and Y. Guo, "Massive hybrid antenna array for millimeter-wave cellular communications," IEEE Wireless Commun., vol. 22, no. 1, pp. 79-87, Feb. 2015.

[2] R. W. Heath, Jr., N. Gonzlez-Prelcic, S. Rangan, W. Roh, and A. M. Sayeed, "An overview of signal processing techniques for millimeter wave MIMO systems," IEEE J. Sel. Topics Signal Process., vol. 10, no. 3, pp. 436-453, Apr. 2016.

[3] W. Roh, et al., "Millimeter-wave beamforming as an enabling technology for $5 \mathrm{G}$ cellular communications: Theoretical feasibility and prototype results," IEEE Commun. Mag., vol. 52, no. 2, pp. 106-113, Feb. 2014.

[4] A. Alkhateeb, J. Mo, N. G. Prelcic, and R. W. Heath Jr., "MIMO precoding and combining solutions for millimeter-wave systems," IEEE Commun. Mag., vol. 52, no. 12, pp. 122-131, Dec. 2014.

[5] O. E. Ayach, S. Rajagopal, S. Abu-Surra, Z. Pi, and R. W. Heath Jr, "Spatially sparse precoding in millimeter wave MIMO systems," IEEE Trans. Wireless Commun., vol. 13, no. 3, pp. 1499-1513, Mar. 2014.

[6] W. Ni and X. D, "Hybrid block diagonalization for massive multiuser MIMO Systems," IEEE Trans. Commun., vol. 64, no. 1, pp. 201-211, Jan. 2016.

[7] C. Kim, T. Kim, and J.-Y. Seol, "Multi-beam transmission diversity with hybrid beamforming for MIMO-OFDM systems," in Proc. IEEE Globecom Workshop - Emerging Tech. for LTE-A and B4G, Atlanta, GA USA, Dec. 13, 2013, pp. 61-65.

[8] L. Dai, X. Gao, J. Quan, S. Han, and C.-L. I, "Near-optimal hybrid analog and digital precoding for downlink mmWave massive MIMO systems," in Proc. IEEE Int. Conf. Commun. (ICC), Jun. 2015, pp. 1334-1339.

[9] J. Zhang, H. Li, X. Huang, and Y. Guo, "User-directed analog beamforming for multiuser millimeter-wave hybrid array systems," submitted in Proc. IEEE Veh. Technol. Conf. (VTC), Jun. 2017.

[10] T. S. Rappaport, et al., "Millimeter wave mobile communications for $5 \mathrm{G}$ cellular: It will work!," IEEE Access, vol. 1, pp. 335-349, 2013. 